\title{
Modified Formula of Estimating Fundamental Frequency of Girder Bridge with Uniform Cross-section
}

\author{
Gao Qing-fei ${ }^{\mathrm{a}}$, Wang Zong-lin ${ }^{\mathrm{b}}$, Liu Yang ${ }^{\mathrm{c}}$, Guo Bin-qiang ${ }^{\mathrm{d}}$ \\ School of Transportation Science and Engineering, Harbin Institute of Technology, Harbin 150090, \\ China \\ aEmail: gaoqingfei_1986@163.com, ${ }^{\text {b} E m a i l: w a n g z o n g l i n @ v i p .163 . c o m, ~}$ \\ 'Email: ly7628@hit.edu.cn, 'Email: guobinqianglove@163.com
}

Keywords: Girder Bridge; Estimation of Fundamental Frequency; Natural Vibration

\begin{abstract}
Based on present research, there is a big difference between the estimation of the first frequency of Girder Bridge obtained by the design code of bridges, issued by department of transportation of China in 2004, and the theoretical value in some cases. To overcome above issue, theoretical analysis combined with numerical simulation was applied to estimate the fundamental frequency of Girder Bridge with uniform cross-section. The effect of the ratio between side span and central span on the estimating fundamental frequency was discussed in detail. Finally, an improved method was proposed to estimate the first frequency of Girder Bridge, and the effectiveness of the proposed method was verified.
\end{abstract}

\section{Introduction}

Most bridges are designed using a static analysis, adjusted by the impact factor which is a function of the natural frequency [1]. According to General Code for Design of Highway Bridges and Culverts, the impact factor of simple-supported girder bridge is bound up with the first flexural frequency of the bridge while it is related to the first two natural frequencies for continuous girder bridge. Thus, the estimated accuracy of natural frequencies is particularly significant.

In the above-mentioned Code, estimated formulas of simply supported girder bridge and continuous girder bridge are suggested as follows [2].

For simply supported girder bridge,

$$
f_{1}=\frac{\pi}{2 l^{2}} \sqrt{\frac{E I_{c}}{m_{c}}} .
$$

For continuous girder bridge,

$$
f_{1}=\frac{13.616}{2 \pi l^{2}} \sqrt{\frac{E I_{c}}{m_{c}}}, \quad f_{2}=\frac{23.651}{2 \pi l^{2}} \sqrt{\frac{E I_{c}}{m_{c}}} .
$$

Where, $f_{1}$ - the first natural frequency, $f_{2}$ - the second natural frequency, $E$ - Young's modulus, $I_{c}$ - second moment of area of mid-span cross-section, $m_{c}$ - mass of mid-span per unit length, and $l$ - span length.

Obviously, the existing estimated formulas have the following defects:

- For multi-spans continuous girder bridge, the meaning and selection of span length $l$ are not clear (the length of central span or side span).

- For multi-spans continuous girder bridge, the number of spans and the ratio of side span and central span are not considered in formula (2).

Shortly, it has an urgent need to study the influence of every parameter and to get a more rational formula of estimating fundamental frequency of Girder Bridge with uniform cross-section. 


\section{Theoretical Analysis}

Based on mechanical concepts, simply supported girder bridge is statically determinate structure while continuous girder bridge is statically indeterminate structure. In this section we develop the equation governing the transverse vibration of a straight girder without damping subjected to external force. D' Alembert principle, Lagrange equation, and Hamilton principle were applied to found the equation of girder bridge vibration [3].

Fig.1a shows such a girder with flexural $E I(x)$ and mass $m(x)$ per unit length, both of which may vary with position $x$. The external forces $p(x, t)$, which may vary with position and time, cause motion of the girder described by the transverse displacement $y(x, t)($ Fig.1b). The equation of motion to be developed will be valid for support conditions other than the simple supports shown and for girders with intermediate supports.

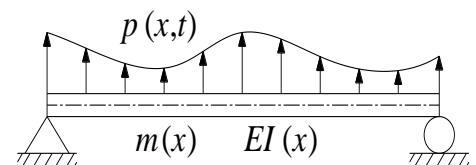

(a) girder and applied force

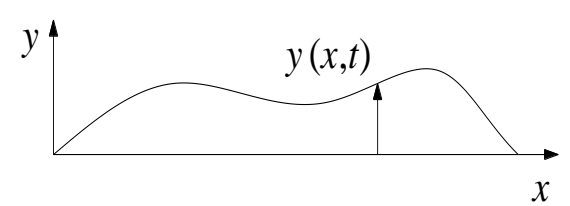

(b) displacement

Fig. 1 System with distributed mass and elasticity

$$
m(x) \ddot{y}+\frac{\partial^{2}}{\partial x^{2}}\left[E I(x) \frac{\partial^{2} y}{\partial x^{2}}\right]=p(x, t) .
$$

\subsection{Simply supported girder}

Setting $p(x, t)=0$ gives the differential equation governing free vibration of the system, which for systems with constant $E I(x)$ and $m(x)$ specializes to

$$
m y+E I \frac{\partial^{4} y}{\partial x^{4}}=0 \text {. }
$$

We attempt a solution of the form

$$
y(x, t)=\varphi(x) q(t) .
$$

Substituting Eq.(5) in Eq.(4) gives

$$
\frac{m}{E I} \frac{\ddot{q}(t)}{q(t)}+\frac{\varphi^{(4)}(x)}{\varphi(x)}=0 .
$$

The first part of equation (6) is a function of $t$ only and the second part depends on $x$. For equation (6) to be valid for all values of $x$ and $t$, the two parts must therefor be constant.

$$
\frac{\varphi^{(4)}(x)}{\varphi(x)}=C=\frac{m}{E I} \frac{\ddot{q}(t)}{q(t)} .
$$

Setting $C=\alpha^{4}$, thus equation (7) becomes two ordinary differential equations, one governing the spatial function $\varphi(x)$ and the other governing the time function $q(t)$.

$$
\left\{\begin{array}{l}
\frac{\mathrm{d}^{4} \varphi(x)}{\mathrm{d} x^{4}}-\alpha^{4} \varphi(x)=0 . \\
\ddot{q}(t)+\omega^{2} q(t)=0 .
\end{array}\right.
$$

where

$$
\omega^{2}=\alpha^{4} \frac{E I}{m} \text {. }
$$

Setting $\varphi(x)=B e^{r x}$, substituting which in the first one of equation (8) gives

$$
\left(r^{4}-\alpha^{4}\right) B e^{r x}=0 \text {. }
$$


Then

$$
r_{1,2}= \pm \alpha, \quad r_{3,4}= \pm i \alpha .
$$

Substituting Eq.(11) in $\varphi(x)=B e^{r x}$ leads to

$$
\varphi(x)=B_{1} e^{a x}+B_{2} e^{-a x}+B_{3} e^{i a x}+B_{4} e^{-i o x} .
$$

So

$$
\varphi(x)=A_{1} \sin \omega x+A_{2} \cos \omega x+A_{3} \sinh \omega x+A_{4} \cosh \omega x .
$$

These four constants $A_{i}(i=1,2,3,4)$ depends on the boundary conditions of the girder.

The boundary conditions of simplified Euler-Bernoulli beam shown in Fig.1(a) are listed as follows:

$$
\varphi(0)=0, \varphi^{\prime \prime}(0)=0, \varphi(l)=0, \varphi^{\prime \prime}(l)=0 .
$$

From Eq.(13)

$$
\varphi^{\prime \prime}(x)=\alpha^{2}\left(-A_{1} \sin \omega x-A_{2} \cos \omega x+A_{3} \sinh \omega x+A_{4} \cosh \omega x\right) .
$$

Combining Eqs.(13), (14) and (15), we obtain the frequency equation:

$$
\sin \alpha=0 \text {. }
$$

Hence the solution is

$$
\alpha_{n} l=n \pi(n=1,2,3, \ldots \ldots) \text {. }
$$

Then, from Eqs.(9) and (17)

$$
\omega_{n}=\alpha_{n}^{2} \sqrt{\frac{E I}{m}}=\left(\frac{n \pi}{l}\right)^{2} \sqrt{\frac{E I}{m}} \quad(n=1,2,3, \ldots \ldots) .
$$

And, for the natural frequencies of simplified girder

$$
f_{n}=\frac{\omega_{n}}{2 \pi}=\frac{n^{2} \pi}{2 l^{2}} \sqrt{\frac{E I}{m}} \quad(n=1,2,3, \ldots \ldots) .
$$

\subsection{Continuous girder}

For common continuous girder bridges, the cross-section is uniform when the span of bridge is small and the cross-section is variable when the span of bridge is large. The continuous girder bridges with variable cross-section are more complicated, and it is difficult to analysis by theoretical methods. Then the continuous girder bridges with uniform cross-section are theoretical analyzed in this paper.

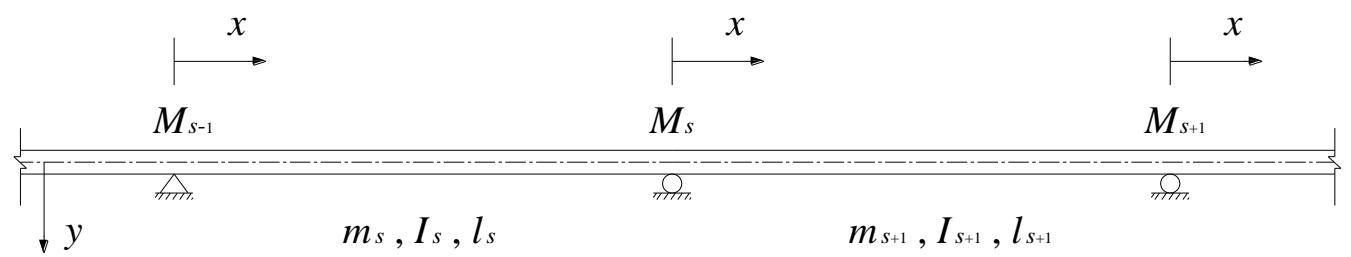

Fig. 2 Symbol conventions of continuous girder

For the continuous girder bridges with uniform cross-section, the distribution of mass and stiffness are equal in every span. The function of the $n$th natural vibration mode in the $s$ th span can be expressed as follows.

$$
\varphi_{n \Sigma}(x)=A_{n \Sigma} \sin \alpha_{n \Sigma} x+B_{n \Sigma} \cos \alpha_{n \Sigma} x+C_{n \Sigma} \sinh \alpha_{n \Sigma} x+D_{n \Sigma} \cosh \alpha_{n \Sigma} x .
$$


These four constants $\left(A_{n s}, B_{n s}, C_{n s}\right.$ and $\left.D_{n s}\right)$ depends on the boundary conditions of the continuous girder. where

$$
\omega_{n}^{2}=\alpha_{n s} \frac{E I_{s}}{m_{s}} \text {. }
$$

The boundary conditions and the continuity of deflection and slope and the compatibility of the forces and moments in the s-span continuous Euler-Bernoulli girder shown in Fig.2 are listed as follows:

$$
\left\{\begin{array}{l}
\varphi_{n s}(0)=0 . \\
\varphi_{n s}\left(l_{s}\right)=0 . \\
\varphi_{n s}^{v}\left(l_{s}\right)=\varphi_{n(s+1)}(0) . \\
E I_{s} \varphi_{n s}^{w}\left(l_{s}\right)=E I_{s+1} \varphi_{n(s+1)}^{w}(0)=-M_{n \Sigma^{*}} \\
\varphi_{n(s+1)}(0)=0 .
\end{array}\right.
$$

From Eq.(20)

$$
\begin{aligned}
& \varphi_{n \Sigma}^{\prime \prime}(x)=\alpha_{n \Sigma}\left(A_{n \Sigma} \cos \alpha_{n \Sigma} x-B_{n \Sigma} \sin \alpha_{n \Sigma} x+C_{n \Sigma} \cosh \alpha_{n \Sigma} x+D_{n \Sigma} \sinh \alpha_{n \Sigma} x\right) \text {. }
\end{aligned}
$$

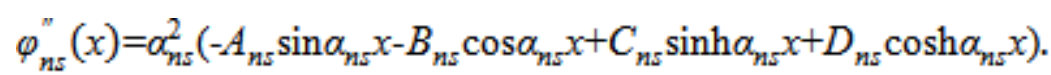

Combining Eqs.(20), (22), (23) and (24), we obtain the frequency equation:

$$
M_{n(s-1)} \frac{H_{n s} l_{s}}{\alpha_{n s} l_{s} I_{s}}-M_{n s}\left[\frac{G_{n s} l_{s}}{\alpha_{n s} I_{s}}+\frac{G_{n(s+1)} l_{s+1}}{\alpha_{n(s+1)} l_{s+1} I_{s+1}}\right]+M_{n(s+1)} \frac{H_{n(s+1)} l_{s+1}}{\alpha_{n(s+1)} l_{s+1} I_{s+1}}=0 .
$$

where

$$
\left.\begin{array}{c}
G_{n \Sigma}=\frac{\cosh \partial_{n \Sigma} l_{n \Sigma}}{\sinh \partial_{n \Sigma} l_{n \Sigma}}-\frac{\cos \partial_{n \Sigma} l_{n \Sigma}}{\sin \partial_{n s} l_{n \Sigma}} \\
H_{n \Sigma}=\frac{1}{\sinh \partial_{n \Sigma} l_{n \Sigma}}-\frac{1}{\sin \partial_{n \Sigma} l_{n \Sigma}}
\end{array}\right\}
$$

From equation (25), A equation can be gained in every support of the continuous girder bridges. And all these equations can be listed as a group. According to set the coefficient determinant of $\{M\}$ as zero, natural frequency equations can be obtained.

For common continuous girder bridges transferred from simply supported girder bridges, the distribution of mass and stiffness are equal in every span. Then the frequency equations can be listed as follows.

1) Two-spans continuous girder:

$G_{n 1}+G_{n 2}=0$.

2) Three-spans continuous girder:

$$
4 G_{n \Sigma}^{2}-H_{n \Sigma}^{2}=0 \quad(s=1,2,3) \text {. }
$$

3) Four-spans continuous girder:

$$
2 G_{n \Sigma}^{2}-H_{n \Sigma}^{2}=0 \quad(s=1,2,3,4) \text {. }
$$

\section{Parametric Study}

As we know, the natural frequencies reflect the distribution of the mass and stiffness of the bridge. The influence of the natural frequencies of Girder Bridge with uniform cross-section consists of the following parameters: the number of spans, the length of central span, the ratio of side span and central span, the properties of mid-span cross-section, and the material properties. 
Taking common continuous girder bridges transferred from simply supported girder as an example, finite element models were made using ANSYS.

Symbol account: $k$ - the ratio of side span and central span, $\lambda-$ the fundamental frequency ratio of bridge with unequal span to bridge with equal span which equal to central span.

Table 1 Physical parameters of finite element model

\begin{tabular}{cc||cc}
\hline Physical parameters & Value & Physical parameters & Value \\
\hline Length of main span & $l=40 \mathrm{~m}$ & Height of section & $h=2.00 \mathrm{~m}$ \\
Area of section & $A=1.3017 \mathrm{~m}^{2}$ & Density & $\rho=2600 \mathrm{~kg} / \mathrm{m}^{3}$ \\
Moment of inertia & $I x=0.6346 \mathrm{~m}^{4}$ & Modulus of elasticity & $E=3.45 \mathrm{e} 10 \mathrm{~Pa}$ \\
\hline
\end{tabular}

Table 2 Vertical natural frequency of Girder Bridge with constant section $(\mathrm{Hz})$

\begin{tabular}{cccccc}
\hline Vertical & Simply supported & \multicolumn{4}{c}{ Continuous girder bridges } \\
\cline { 3 - 6 } mode order & girder bridges & Two-spans & Three-spans & Four-spans & Five-spans \\
\hline 1 & $\mathbf{2 . 4 9 3}$ & $\mathbf{2 . 4 9 3}$ & $\mathbf{2 . 4 9 3}$ & $\mathbf{2 . 4 9 3}$ & $\mathbf{2 . 4 9 3}$ \\
2 & $\mathbf{9 . 9 2 8}$ & 3.894 & 3.195 & 2.908 & 2.766 \\
3 & & $\mathbf{9 . 9 2 8}$ & 4.664 & 3.894 & 3.458 \\
4 & & & $\mathbf{9 . 9 2 8}$ & 5.030 & 4.355 \\
5 & & & & $\mathbf{9 . 9 2 8}$ & 5.229 \\
6 & & & & & $\mathbf{9 . 9 2 8}$ \\
\hline
\end{tabular}

Table 3 Fundamental frequency of Girder Bridge with constant section

\begin{tabular}{ccc||ccc||ccc}
\hline Bridge $(\mathrm{m})$ & $k$ & $f_{1}(\mathrm{~Hz})$ & Bridge $(\mathrm{m})$ & $k$ & $f_{1}(\mathrm{~Hz})$ & Bridge $(\mathrm{m})$ & $k$ & $f_{1}(\mathrm{~Hz})$ \\
\hline $20+40+20$ & 0.500 & 3.894 & $20+35+20$ & 0.571 & 4.883 & $20+30+20$ & 0.667 & 6.251 \\
$25+40+25$ & 0.625 & 3.620 & $25+35+25$ & 0.714 & 4.438 & $25+30+25$ & 0.833 & 5.415 \\
$30+40+30$ & 0.750 & 3.302 & $30+35+30$ & 0.857 & 3.881 & $30+30+30$ & 1.000 & 4.427 \\
$35+40+35$ & 0.875 & 2.914 & $35+35+35$ & 1.000 & 3.255 & $20+25+20$ & 0.800 & 8.053 \\
$40+40+40$ & 1.000 & 2.493 & & & & $25+25+25$ & 1.000 & 6.368 \\
\hline
\end{tabular}

Table $4 k$ and $\lambda$ of Girder Bridge with constant section

\begin{tabular}{llllllllllll}
\hline$k$ & 0.500 & 0.571 & 0.625 & 0.667 & 0.714 & 0.750 & 0.800 & 0.833 & 0.857 & 0.875 & 1.000 \\
\hline$\lambda$ & 1.562 & 1.500 & 1.452 & 1.412 & 1.363 & 1.325 & 1.265 & 1.223 & 1.192 & 1.169 & 1.000 \\
\hline
\end{tabular}

\section{Results and Discussion}

Table 2 shows that the fundamental frequency of continuous girder bridge is the same as the simplified supported girder bridge, the length of which is equal to the main span of the continuous girder bridge. In other words, the number of spans has no influence on the fundamental frequency of Girder Bridge with equal span and constant section.

Table 3 shows the fundamental frequency of various Girder Bridge with different span arrangement. As we can see, the fundamental frequency decreases with the increasing of the main-span length when the side-span length is constant. And the fundamental frequency increases with the decreasing of the side-span length when the main-span length is constant.

Table 4 shows the influence by introducing two parameters $(k$ and $\lambda)$. The modified factor $\lambda$ decreases with the increasing of the ratio $k$. A regression equation between $k$ and $\lambda$ can be found by statistical analysis as follows.

$$
\lambda=-0.675 k^{2}-0.113 k+1.787(0<k \leq 1.0)\left(R^{2}=0.9999 \approx 1.0\right) .
$$

And now

$$
f_{1}=\lambda \frac{\pi}{2 l^{2}} \sqrt{\frac{E I_{c}}{m_{c}}}
$$




\section{Conclusions}

The theoretical analysis combined with numerical simulation was applied to estimate the fundamental frequency of Girder Bridge with uniform cross-section in this paper. The results showed that the number of spans has no effect on the estimation of fundamental frequency of Girder Bridge with equal span and constant section. Based on this result, a improved estimation of the first frequency of Girder Bridge was proposed and the effectiveness was verified.

\section{References}

[1] T.J. Memory, D.P. Thambiratnam, G.H. Brameld, Free vibration analysis of bridges, J. Engineering Structure. 17(1995) 705-713.

[2] Department of the transport of P R China, General code for design of highway bridges and culverts, S. China Communication Press, Beijing, 2004, pp. 83-84.

[3] Anil K. Chopra, Dynamics of Structures - Theory and Applications to Earthquake Engineering (third edition), M. Tsinghua University Press, Beijing, 2009, pp. 629-630. 